\title{
Las clínicas jurídicas como actores claves en la defensa de los derechos de las víctimas en el posacuerdo*
}

\begin{abstract}
Mayra Alejandra García Ramírez ${ }^{* *}$
Resumen. A través de este artículo se pretende identificar cuál es el papel de las clínicas jurídicas de interés público en relación con la defensa de los Derechos Humanos (DDHH) de las víctimas en el marco del posacuerdo. El punto de partida son los estudios sobre la función de la educación clínica como práctica transformadora y la necesidad de establecer el rol de estas, en la protección de sus derechos sin que ello implique sustituir el papel del Estado. Para ello se ha propuesto, en primer lugar, revisar ¿qué se entiende por defensa de derechos humanos de las víctimas?, comprendiendo el alcance, la finalidad y las formas que la constituyen. En segundo lugar, efectuar un análisis sobre los retos que afronta Colombia en la reparación y amparo de sus facultades, con el propósito de acercarnos a una propuesta de aquello que debería ser el trabajo clínico bajo su conservación y, finalmente, compartir algunas ideas y experiencias de la labor de la Clínica Jurídica en Derechos Humanos de la Universidad Santiago de Cali (USC).
\end{abstract}

Palabras claves: Clínicas jurídicas, defensa, víctimas, posacuerdo, derechos humanos.

\footnotetext{
*Ponencia presentada en el marco del VII Encuentro Nacional de Clínicas Jurídicas de interés público: El papel de las Clínicas Jurídicas de interés público en la construcción de paz en el posacuerdo; subtema: Función de las Clínicas Jurídicas en la defensa de los derechos de las víctimas. Ibagué, junio de 2017.

** Abogada, magíster en Derecho de la Universidad Santiago de Cali. Coordinadora de la Clínica Jurídica en Derechos Humanos de la Universidad Santiago de Cali y docente tiempo completo de las asignaturas Derechos Humanos y Justicia Transicional. Integrante del grupo de investigación GICPODERI de la Universidad Santiago de Cali.
} 


\section{Introducción}

El presente artículo intenta responder el siguiente problema: ¿Cuál es el papel que deben desempeñar las clínicas jurídicas de interés público en relación con la defensa de los derechos de las víctimas en el posacuerdo? Para empezar, se concede una aproximación a qué se entiende por defensa de derechos humanos de las víctimas y se señalan algunos ejemplos que la establecen. Posteriormente, se enseña el panorama actual en relación con las víctimas del conflicto armado del país, para explicar cuáles son los retos que debe afrontar el Estado colombiano, con el fin de garantizar su reparación en una etapa de posacuerdo. A la vez, a partir de las experiencias y aprendizajes obtenidos en la Clínica Jurídica en Derechos Humanos de la Universidad Santiago de Cali se presenta una propuesta para dar respuesta a la pregunta planteada en principio.

La tesis central radica en que la función de las clínicas jurídicas es complementaria a las acciones desarrolladas por el Estado y por las organizaciones defensoras de derechos humanos; que, en ningún caso es posible sustituir el papel del Estado y que es necesario empoderar a las víctimas no solo para reclamar la aplicación de sus derechos, sino también, para reintegrarlas a la sociedad civil y establecer el cese de su condición.

La estructura de la ponencia responde a la necesidad de comprender el contenido y el alcance de aquello que significa defender los derechos de las víctimas, más allá de las intervenciones administrativas y judiciales que en el marco del litigio estratégico puedan desarrollarse. En este sentido, la identificación de actividades de formación, de pedagogía, la investigación en derechos humanos, el empoderamiento y el acompañamiento a esta población, hará sostenible la reparación y la reincorporación a la sociedad en una fase de posacuerdo.

La autora agradece el apoyo de la docente investigadora adscrita a la Clínica Jurídica en Derechos Humanos de la Universidad Santiago de Cali, doctora Liliana Ambuila Valencia, en la elaboración y revisión de este artículo.

\section{Objetivos}

\section{Objetivo general}

- Establecer cuál es el papel que deben desempeñar las clínicas jurídicas de interés público en relación con la defensa de los derechos de las víctimas en el posacuerdo. 


\section{Objetivos específicos}

- Determinar el contenido y el alcance del concepto de defensa en el marco del ejercicio de los derechos de las víctimas.

- Analizar cuáles son los retos que enfrenta el Estado colombiano, en relación con la reparación de las víctimas del conflicto armado interno.

- Presentar una propuesta acerca de qué debería ser el trabajo clínico en un contexto de posacuerdo, con respecto a la defensa de los derechos de las víctimas.

\section{Metodología}

El presente trabajo se plantea desde una perspectiva analítica sobre el papel de las clínicas jurídicas de interés público, en relación con la defensa de los derechos humanos de las víctimas en el posacuerdo. Al finalizar, se presentan algunas ideas sobre el papel que deberían desempeñar, a partir de la experiencia de la Clínica Jurídica en Derechos Humanos de la USC.

\section{Defender los derechos de las víctimas: contenido y finalidad}

Pensar en cuál es la función que deben desempeñar las clínicas jurídicas de interés público frente a la defensa de los derechos de las víctimas en el posacuerdo implica entender su contenido y qué mecanismo o formas existen para ello. Según señala el Diccionario de la Real Academia de la Lengua Española (DRAE, 2017) defender es sinónimo de amparar, librar, proteger, mantener o sostener algo contra el dictamen ajeno. La defensa de los derechos de las víctimas podría ser entendida, como toda aquella acción encaminada a su conservación en cualquier contexto.

Una definición más amplia la proporciona el campo de los Derechos $\mathrm{Hu}-$ manos, bajo la cual entenderemos su protección como una modalidad de su tutela. En este sentido, la defensa de los derechos humanos constituye uno de los instrumentos de trabajo crecientemente utilizados por diferentes organizaciones, orientados al desarrollo de los intereses y al empoderamiento de los sectores sociales con mayor grado de vulnerabilidad y de exclusión social y política (Diccionario de Acción Humanitaria y Cooperación al Desarrollo, 2017).

Si revisamos las actividades desarrolladas por personas u organizaciones defensoras de derechos humanos encontramos una multiplicidad de acciones que van desde la defensa legal de las víctimas, las campañas educativas y la propuesta de nuevas leyes, hasta la investigación y documentación sobre vio- 
laciones de los derechos humanos (Diccionario de Acción Humanitaria y Cooperación al Desarrollo, 2017).

Hasta ahora, solo se ha intentado una aproximación conceptual respecto de qué se entiende por defensa de derechos en el campo de los derechos humanos. Esta, no solo ofrece una definición adecuada, sino que también concede ejemplos de las diversas actividades desarrolladas por sus defensores ${ }^{1}$. Dentro de ellas encontramos las siguientes²:

Ejercicio de acciones jurídicas locales, nacionales e internacionales: Una de las más recurrentes es por vía administrativa o judicial. Este camino es adecuado cuando se ha producido un daño y se busca la obtención de reparación. Las acciones pueden estar acompañadas de otro tipo de actividades tendientes a lograr verdad, justicia, reparación y garantías de no repetición.

Investigación y difusión sobre violaciones de Derechos Humanos ${ }^{3}$ : Los defensores de los derechos humanos también investigan, documentan y difunden casos de violaciones de los mismos, como estrategia de promoción y resguardo, pues a través de este mecanismo ejercen presión política o social y ubican el debate en el escenario público.

Incidencia institucional para lograr el cumplimiento de las obligaciones derivadas de los tratados internacionales sobre Derechos Humanos: En la organización del Estado son diversas las entidades que poseen competencias directas en relación con la promoción y protección de los derechos humanos. Por eso, el ejercicio de incidencia con cada una de ellas, también constituye un campo de acción muy importante en el marco de su defensa.

\footnotetext{
${ }^{1}$ Se ha extendido el empleo de la expresión Defensor de los derechos humanos a raíz de la aprobación de la Declaración sobre los Defensores de los Derechos Humanos en 1998. Hasta entonces, términos como el de activista, profesional, agente o supervisor de los derechos humanos habían sido los más comunes. Se considera que Defensor de los derechos humanos es más pertinente y útil. Ver: Folleto informativo No. 29 Los Defensores de los Derechos Humanos: Protección del Derecho a Defender los Derechos Humanos. Disponible en: http://www.ohchr.org/Documents/Publications/FactSheet29sp.pdf 2 "Se usa la expresión Defensor de los derechos humanos para describir a la persona que, individualmente o junto con otras, se esfuerza en promover o proteger esos derechos. Se les conoce sobre todo por lo que hacen, y la mejor forma de explicar lo que son consiste en describir sus actividades y algunos de los contextos en que actúan. Los ejemplos que se ofrecen de sus actividades no constituyen una lista exhaustiva". Ver: Folleto informativo No. 29 -Los Defensores de los Derechos Humanos: Protección del Derecho a Defender los Derechos Humanos. Disponible en: http://www.ohchr.org/Documents/ Publications/FactSheet29sp.pdf

${ }^{3}$ El activismo en redes sociales resulta ser una estrategia de difusión sobre violaciones a los derechos humanos. Algunos ejemplos son change.org: https://www.change.org/ y AVAAZ: https://secure.avaaz. org/page/es/
} 
Educación y capacitación en materia de derechos humanos: Uno de los retos más grandes en el ejercicio de defensa de los derechos humanos es lograr que todos los sectores, instituciones, sociedad civil y víctimas, cuenten con sus conocimientos mínimos. Esto implica garantizar que los funcionarios adopten protocolos de atención bajo parámetros de enfoque diferencial, que conozcan el alcance normativo, e incluso, institucional de la entidad que representan y la importancia de la no revictimización por parte de las instituciones y de la sociedad civil. De la misma manera, que empoderen a los afectados para exigir del Estado las garantías correspondientes (Oficina del Alto Comisionado de las Naciones Unidas para los Derechos Humanos, 2017).

Con fundamento en lo anterior defender los derechos de las víctimas no solo incluye las acciones administrativas o judiciales a través de las cuales se pretende evitar un perjuicio irremediable, reparar un daño causado o impedir que se produzcan nuevas vulneraciones de derechos. También involucra capacitarlas sobre la ruta que deben seguir para obtener las medidas de asistencia, atención y reparación, con el propósito de identificar cuál es su posición en el ámbito social, jurídico y político del país. A través de la educación se transforma la visión tradicional de que ellas necesitan representantes para el ejercicio y la garantía de sus derechos.

\section{Retos que afronta Colombia en la reparación de los derechos de las víctimas del conflicto armado}

De acuerdo con lo expresado hasta el momento, la defensa de los derechos de las víctimas tiene por objeto promover y reparar. En ese sentido, antes de indicar cuál es el papel que las clínicas jurídicas de interés público deben asumir frente a esta situación, es importante revisar cuáles son los retos que afronta el Estado colombiano en relación con la promoción y la reparación de las víctimas del conflicto armado. Esto permite dimensionar la magnitud del problema e identificar dentro del marco de las competencias de las clínicas jurídicas, cuál puede ser el mayor aporte que se efectúe con respecto a este tema.

Se identifican varios retos en la promoción de derechos de las víctimas del conflicto armado en Colombia; uno es que el Estado debe propender porque ellas conozcan sus obligaciones y derechos derivados de la vulneración de estos últimos.

En ese hilo de argumentación, también será un desafío para el país lograr que se conozca la verdad sobre lo ocurrido durante estos más de cincuenta años de conflicto armado interno, es decir, que sepan qué, cuándo y dónde sucedió y 
quiénes financiaron y facilitaron (políticos, empresas, particulares) la comisión de hechos victimizantes. Lograrlo devolverá la confianza perdida en la institución, contribuirá a fomentar el respeto irrestricto de los derechos humanos y ayudará a prevenir la repetición de los hechos victimizantes.

Frente a la reparación de las víctimas del conflicto armado en Colombia, "el reto primordial radica en la capacidad del Estado para diseñar un procedimiento expedito y eficaz, que restituya a la víctima a las condiciones que tenía antes de la ocurrencia del hecho victimizante" (Garay Salamanca \& Vargas Valencia, 2012, p. 50-51), en medio de la vigencia del conflicto, en el que no ha existido espacio para la transición.

Desde la reparación transformadora, el desafío no es solo reintegrarla a las condiciones que tenía antes del hecho victimizante, sino también, mejorar su condición; pues en muchos casos, regresar a las víctimas a su estado anterior es revictimizarlas, porque su condición ya era precaria en el pasado.

Es indudable que, en los últimos veinte años, el Estado haya expedido una serie de normas a través de las cuales se han consagrado medidas de reparación en el marco del conflicto armado. Así encontramos que el primer referente normativo es la Ley 418 de 1997, a través de la cual se establecen mecanismos de atención y asistencia para las víctimas de violencia política. Posteriormente se expide la Ley 975 de 2005 o Ley de Justicia y Paz, por medio de la cual se busca reparar de manera individual a todas las víctimas de miembros de grupos armados organizados al margen de la ley. Es la primera vez que se señala un procedimiento de reparación por vía administrativa, el cual fue reglamentado a través del Decreto 1290 de 2005. Además de establecer este procedimiento, los hechos victimizantes y los montos a otorgar por concepto de indemnización, se incluyeron medidas de rehabilitación y de satisfacción para las víctimas del conflicto armado, lo cual manifiesta un avance significativo en relación con la reparación integral. Finalmente, la Ley de Víctimas y Restitución de Tierras —en adelante Ley 1448 de 2011 - es aquella que logra recoger en un mismo texto normativo las demandas de asistencia, atención y reparación para quienes sufren las consecuencias del conflicto armado.

A través de la Ley 1448 de 2011 y sus decretos reglamentarios ${ }^{4}$, no solo se amplía el concepto de víctima, sino que, se crean entidades especializadas para la reparación integral y para la restitución de tierras. Se establece un margen de

\footnotetext{
${ }^{4}$ Entre otros, ver Decreto 4800 de 2011, Decreto 4633 y 4635 de 2011 y Decreto 4829 de 2011 modificado por el Decreto 1071 de 2015.
} 
tiempo en el cual todas deben ser atendidas y se concibe un amplio desarrollo en relación con las medidas de rehabilitación y satisfacción.

En la actualidad, después de la firma del acuerdo final para la terminación del conflicto entre el Gobierno de Colombia y la guerrilla de las FARC-EP, se han expedido diferentes instrumentos jurídicos, a través de los cuales se incluyen mecanismos judiciales y extrajudiciales para hacer efectiva la reincorporación de los miembros de la guerrilla a la vida civil y se proponen mecanismos de reparación y garantías de no repetición. A través del acto legislativo 01 de 2017 se crea el Sistema Integral de Verdad, Justicia, Reparación y Garantías de no Repetición, que está conformado por la Comisión para el Esclarecimiento de la Verdad, la Convivencia y la No Repetición, la Unidad para la Búsqueda de Personas dadas por Desaparecidas en el contexto y en razón el conflicto armado, la Jurisdicción Especial para la Paz y las medidas de reparación integral para la construcción de paz y garantías de no repetición. Aunque aún son numerosas las preguntas que existen sobre la estructura final, lo actual vislumbra cambios significativos en las formas de reparación y en los mecanismos administrativos, judiciales y extrajudiciales establecidos para ello, razón por la cual, se convierte en un reto el conocimiento que de estos instrumentos tengamos todos.

Ante la necesidad de identificar, esclarecer, reconocer y responder a la magnitud de los daños materiales y morales generados como consecuencia del conflicto armado, es necesario saber que, según los datos reportados por la Unidad para la Atención y Reparación Integral a las Víctimas (UARIV), en Colombia existen 8405265 víctimas del conflicto armado, de quienes 7159144 corresponden a desplazamiento forzado (Unidad de víctimas, 2017). A simple vista su situación en nuestro contexto es una tragedia humanitaria y para clarificarla, se recurre a los casos utilizados por el Centro de Memoria Histórica. Pensar en esta población como tamaño de localidades resulta más ejemplificador: si ubicamos a todas las víctimas del conflicto en una misma ciudad, estas podrían ser del tamaño de Bogotá o, quizá, más grande (Centro Nacional de Memoria Histórica, 2013).

Lo anterior indica que el $86 \%$ de las víctimas que ha dejado el conflicto colombiano corresponden a desplazamiento forzado, situación que nos ubica en el primer puesto de los países con mayor número en este plano (ACNUR, Agencia de la ONU para los refugiados, 2017) y el segundo después de Afganistán con mayor cifra por minas antipersonales: 10.990 (Colombia Humanum, 2017). 
El desplazamiento forzado es una de las mayores tragedias humanitarias, acarrea toda suerte de rupturas y pérdidas y supone una violación simultánea, múltiple y continua de un extenso listado de derechos (Ceballos Bedoya, 2012). Si bien, no todos los desplazados forzados eran pobres antes de los hechos de violencia, lo cierto es que los niveles de pobreza en relación con las víctimas de desplazamiento forzado aumentaron. Del $50 \%$ pasaron al $97 \%$; los niveles de indigencia pasaron del $23 \%$ al $80 \%$ (Garay Salamanca et al., 2009). El reto que tiene Colombia frente a la reparación es enorme, pues no solo debemos remediar los daños ya causados, sino también, desarrollar estrategias para garantizar que no exista otra víctima de los mismos hechos, ni que las existentes vuelvan a serlo. "El desafío está en reconstruir proyectos de vida y tejidos sociales que se han roto con el desplazamiento" (Garay Salamanca \& Vargas Valencia, 2012, p. 50-51).

Colombia fue el primer país del mundo en iniciar procesos de reparación en medio del conflicto armado, lo cual supone la revisión permanente de las condiciones de seguridad, en cada una de las intervenciones desarrolladas por las instituciones que generan medidas que garanticen la no repetición. La lucha que afronta el Estado pasa por establecer las medidas de reparación adecuadas, el diseño de procedimientos expeditos, el deber de preservar la memoria y de realizar procesos de formación y promoción sobre la necesidad de respetar y defender los derechos humanos, no solo en contextos de conflictos armados, sino también, en épocas de transición y de paz.

\section{El papel de las clínicas jurídicas frente a la defensa de los derechos de las víctimas}

\section{1. ¿Para qué la enseñanza clínica del Derecho?}

Antes de aproximarnos a algunas ideas sobre el rol que deben desempeñar las clínicas jurídicas en relación con la defensa de los derechos de las víctimas en una fase del posacuerdo, es importante efectuar una revisión de los estudios sobre el origen y la evolución de la enseñanza del método clínico. Lo anterior, porque la estructuración de la clínica jurídica como práctica trasformadora del derecho se convierte en el punto de partida para identificar ese papel, entendiendo su razón de ser y las diferentes acciones que constituyen modalidades de salvaguardia en el marco de los derechos de las víctimas.

Algunos estudios sobre el origen de las clínicas jurídicas señalan que la educación clínica surge en gran medida como reacción a la educación jurídica tradicional, enciclopedista, memorista y poco práctica (Courtis, 2007) en 
la que a los estudiantes de las facultades de Derecho les enseñan la norma y cuáles son las etapas del proceso, la jurisprudencia y la doctrina, las escuelas y los teóricos.

Por lo anterior se identifica "una característica predominante en la enseñanza del Derecho en América Latina y es la de la existencia de un énfasis excesivo en la memorización, en desmedro del desarrollo de la capacidad de argumentación" (González Morales, 2004, p. 11).

Así pues, su método tradicional plantea la idea de que el único objeto de estudio lo constituyen las normas jurídicas y el razonamiento, conforme al cual los conflictos que se susciten en una sociedad deben resolverse solo de acuerdo con las normas jurídicas que estén vigentes en ese momento. De lo contrario, la resolución sería arbitraria (González Morales, 2004). De seguir en este método, la enseñanza del Derecho solo es posible proyectarla a través de estudios descriptivos y reduccionistas. Este no solo reduce el objeto de estudio e investigación del mismo, sino que:

... Por la misma formación tradicional que han recibido la mayoría de los jueces, las características de la argumentación que ellos esperan de los abogados no se asocia con un sentido fuerte del concepto. Para romper el círculo vicioso se requiere, entonces, modificar las modalidades de evaluación en la enseñanza jurídica, que en definitiva terminará fortaleciendo una capacidad de argumentación genuina tanto en las presentaciones escritas y las alegaciones de los abogados, como en las sentencias de los jueces. (González Morales, 2004, p. 180)

Ahora bien, respecto de los países del sistema del Common Law, la forma en que se lleva adelante la enseñanza del Derecho tiende a ir más por la vía de entregar al alumno las destrezas que le permitan analizar, evaluar, enunciar y comparar situaciones reales concretas, que ser capaz de seleccionar los contenidos de las normas jurídicas en las clases y repetir aquello que dice el educador en sus exámenes. Los profesores no son profesionales, en el sentido en que su tarea se parece más a un hobby que a una actividad que demanda intensa dedicación (González Morales, 2004).

Para los docentes del Common Law, la enseñanza del Derecho es una tarea subordinada a la principal, que es, en general, la de trabajar como abogado o juez. Puesto que, el estudio de casos implica el enfrentamiento con el mundo real, abstracción, interpretación y análisis de las normas constitucionales y legales sobre la materia. El estudiante, entonces, se acerca a las actividades desarrolladas por los jueces y abogados. 
La propuesta clínica surge de la necesidad de romper los paradigmas tradicionales en la enseñanza del Derecho y parte en principio, del estudio de casos para acercarse a los roles de abogado o juez, pero, posteriormente, vincula todo tipo de ejercicios profesionales, incluso, desde las funciones legislativas.

\subsection{Propuesta del trabajo clínico a partir de las experiencias de la Clínica} Jurídica en DDHH de la USC

En el desarrollo y la evolución que han adquirido las clínicas jurídicas encontramos un rasgo característico y es su trabajo en litigio estratégico de interés público. Colombia no ha sido ajena a esta evolución, pues ha encontrado en él una posibilidad de generar mayor impacto en la protección y salvaguarda de los derechos humanos.

A través de la transformación de la enseñanza del Derecho, en América Latina y en Colombia, la propuesta clínica ha permitido que las facultades de Derecho realicen litigio estratégico de interés público en derechos humanos, que consiste en la selección y presentación de un caso ante los tribunales nacionales o internacionales, como un medio para defender y promover los derechos humanos (Pelletier Quiñones, 2012).

"A pesar de que el objeto del litigio de impacto en derechos humanos o litigio de interés público es obtener una decisión favorable que produzca efectos jurídicos y sociales, el hecho de no ganar el caso no significa una derrota" (Pelletier Quiñones, 2012, p. 320), porque la presentación y socialización de este, permiten evidenciar una problemática sobre la cual el Estado no ha intervenido o la manera en que lo ha hecho resulta poco efectiva o eficaz. Generar presión política nacional o internacional a partir de la visibilización del caso, incluso, que la sociedad opine, tome partido o conciencia sobre el particular, resulta también ganancia.

Las clínicas jurídicas han entendido que efectuar litigio de interés público resulta beneficioso en tanto requiera menor desgaste de recursos físicos y económicos y el impacto de los resultados podría beneficiar a un número indeterminado de personas o a una comunidad específica de la sociedad.

Bajo esta lógica y frente a las necesidades actuales del país, la propuesta en el marco de la defensa de los derechos humanos tendrá que estar unida inescindiblemente al contexto nacional, frente al desarrollo e implementación de los acuerdos (fase de posacuerdos) y también a la consolidación de la paz.

La Clínica Jurídica de la Universidad Santiago de Cali propuso intervenir las líneas misionales (docencia, investigación y extensión) con el objeto de 
aportar a la promoción y la reparación a las víctimas como acciones derivadas de la defensa. Para ello, desde la docencia, a través de cursos electivos se introdujo la cátedra de Justicia Transicional y Litigio Estratégico en Derechos Humanos. Puesto que promocionar los derechos de las víctimas debe partir de la educación sobre la genealogía del conflicto, el concepto y alcance de la justicia transicional, las experiencias comparadas y el actual proceso de paz. Por su parte litigar en el área de los derechos humanos requiere no solo adquirir los conocimientos teóricos y conceptuales relacionados con el tema, sino que, implica conocer cómo documentar un caso en derechos humanos, cuáles son las estrategias de litigio y sus mecanismos de promoción, protección y reparación.

Desde la investigación, la Clínica Jurídica interviene en la presentación de amicus y sus proyectos aprobados por la Dirección General de Investigaciones de la Universidad están relacionados con la evaluación y presentación de propuestas en torno a la reparación de víctimas, la restitución de tierras, las experiencias comparadas respecto a la Justicia Transicional, la Comisión de la Verdad, la Memoria Histórica.

En la labor de extensión, la Clínica ha propuesto la realización de jornadas de actualización, diplomados (educación continua), seguimiento a políticas públicas, entre otros; que permitan cumplir con la función de educar a nuestros egresados y a la población local y regional sobre las normas, instrumentos y el proceso actual de transición.

No obstante, ese ejercicio ha permitido evidenciar dos aspectos importantes que facilitan una formulación de propuesta de trabajo clínico encaminado a la defensa de los derechos de las víctimas del conflicto armado.

Por un lado, desde el ejercicio de acciones que generan un vínculo externo (todas las actividades de extensión) es evidente el rechazo por parte de la sociedad civil a la reintegración social de actores del conflicto que se están desmovilizando, pero paradójicamente, también frente a las víctimas sumado al desconocimiento sobre aspectos generales de la implementación de los acuerdos.

Y, por otro, de cara a lo interno, el sistema nacional de atención, asistencia y reparación a las víctimas, el marco jurídico para la paz y la justicia de transición en la actualidad son tan amplios en términos normativos y jurisprudencial, que casi crean una jurisdicción sobre la cual, sin profesionales que trabajen el tema, resulta insuficiente que la orientación a estas personas en los consultorios jurídicos se limite a la remisión a entidades especializadas.

Construir una propuesta que incluya todos los escenarios de defensa, que tenga en cuenta las dificultades y los retos de reparar a las víctimas en el con- 
texto colombiano y que no pierda de vista la razón de ser de las clínicas jurídicas, no es una tarea menor, pues genera grandes desafíos.

Al atender el objetivo de las Clínicas Jurídicas en derechos humanos, la labor en litigio de interés público y la intención de trabajo experiencial que contribuya en la formación de los estudiantes como futuros abogados sumado al contexto nacional, en el que es necesario asumir la protección de las víctimas del conflicto armado, una propuesta sobre su papel en derechos humanos en relación con la promoción y la defensa de sus derechos de cara al posacuerdo deberá contener:

Litigio de interés público: Presentar las acciones constitucionales o de intervenciones judiciales que permitan mejorar, transformar o proponer ajustes normativos o procedimentales que garanticen la efectividad y cumplimiento de los derechos de las víctimas, que integran las técnicas de litigio estratégico en derechos humanos.

Compartir experiencias: Fomentar los espacios que permitan compartir vivencias entre clínicas para conocer obstáculos, avances, retrocesos, estrategias, áreas de trabajo y problemáticas sobre las cuales se trabaja, incluso, para la construcción de alianzas de cooperación.

Formación: Contribuir desde el concepto de educación continua en estudiantes, egresados, funcionarios públicos, miembros de la rama judicial y sociedad civil, sobre la implementación de los acuerdos, el conocimiento permite derribar mitos.

Investigación: Explorar soluciones y propuestas desde la disciplina de la investigación científica es un rol propio de las instituciones educativas. $\mathrm{Y}$ en el área de los derechos humanos de las víctimas, las clínicas jurídicas deben propender porque se desarrollen, financien y difundan los resultados de este tipo de estudios.

\section{Conclusiones}

- El modelo de clínica surgió con el propósito de transformar la enseñanza tradicional del Derecho a partir de la necesidad de ampliar el objeto de estudio, más allá de memorizar y recitar la norma. Conforme al paso del tiempo, a la evolución de las sociedades, de las instituciones y de sus conflictos, es necesario proponer alternativas creativas para que la enseñanza de esta ciencia tenga la capacidad de transformar realidades nacionales, 
regionales y locales, que implican revisar en forma permanente el contexto territorial sobre el cual actúa.

- Son muchas las necesidades con las que cuentan actualmente las víctimas del conflicto armado en el país. Algunas de ellas pueden resolverse quizá, con un derecho de petición o una acción de tutela, pero el caso a caso, en forma individual y por demanda, no debe ser una función de las clínicas jurídicas, porque no solo desnaturalizamos el rol que desempeñan, sino que también, invadimos funciones de los Consultorios Jurídicos a los cuales llegan todas las demandas de derechos de poblaciones vulnerables que no pueden acceder a un abogado privado.

- Aun cuando admitiéramos que las clínicas jurídicas pueden desempeñar funciones de representación judicial de víctima en víctima, la pregunta que deberíamos formularnos es ¿el rol de las clínicas jurídicas en relación con la defensa de los derechos de las víctimas se cumple saturando a las autoridades administrativas y judiciales con solicitudes que no han podido cumplirse por razones estructurales? Su deber es precisamente identificar cuáles son esas dificultades estructurales y sistemáticas que conllevan a que la realización de los derechos de las víctimas no sea una realidad, y proponer, desde esa indagación, estrategias y alternativas que permitan que todas se beneficien de las mismas.

- Admitir que la labor de las clínicas jurídicas es esencialmente práctica, no desconoce la necesidad de fortalecer aspectos teóricos. Ellas están llamadas a formar en derechos humanos, sobre generalidades, mecanismos de protección y herramientas de defensa y acerca del papel que cada integrante de la sociedad juega a su interior.

- Una de las consecuencias que ha dejado el conflicto fue la imposibilidad de documentar graves violaciones de DDHH y del Derecho Internacional Humanitario (DIH) en zonas afectadas significativamente por este. Desde la labor de investigación, las clínicas jurídicas pueden formular proyectos encaminados a documentar las violaciones de derechos humanos, a proponer alternativas para mejorar la condición de las víctimas y a garantizar que se reincorporen a la sociedad para que en algún momento su condición desaparezca. Estas investigaciones constituyen una forma de hacer memoria, al tiempo que entregan insumos a la institucionalidad para que avancen en su trabajo.

- Pese a que llevamos más de cinco décadas en conflicto armado, un porcentaje significativo de la población colombiana desconoce su origen y 
consecuencias, muchos ni siquiera saben dónde quedan los territorios que mayor afectación han tenido en relación con el número de víctimas. Es labor de la academia difundir conocimientos con respecto a este tema. Las clínicas jurídicas pueden aportar en forma significativa, a través de la inclusión de asignaturas en los programas de pregrado y posgrado, como también, por medio de procesos de capacitación que lleguen a todos los sectores y que puedan establecer criterios diferenciadores para la población que ha sido víctima. Esta es la única forma que tenemos de empoderarlas y acompañarlas; así, les permitimos que conozcan sus derechos y les enseñamos cómo los deben ejercer.

\section{Referencias}

ACNUR Agencia de la ONU para los Refugiados. (2017). Tendencias Globales sobre refugiados y otras personas de interés del ACNUR. Recuperado de http://www.acnur. org/recursos/estadisticas/

Bedoya, M. A. (2012). Protección Diferenciada de Derechos en Colombia. La condición de desplazado como clave de acceso del derecho a la vivienda. En Gallego García, G. M. \& González, M. J. (Ed.), Conflicto armado, justicia y reconciliación (p. 226). Bogotá, Colombia: Siglo del Hombre Editores.

Centro Nacional de Memoria Histórica. (2013). Basta Ya: Colombia: memorias de guerra y dignidad. Bogotá, Colombia: CNMH.

Colombia Humanum. (2017). Recuperado de http://www.humanumcolombia.org/colombia-el-segundo-pais-con-mas-victimas-de-mapmuse-en-el-mundo/

Courtis, C. (2007). La educación clínica como práctica transformadora. Recuperado de file:///C:/Users/lenovo/Downloads/La_ense_anza_Cl_nica_como_pr_ctica_ transformadora\%20(1).pdf

Diccionario de Accción Humanitaria y Cooperación al Desarrollo. (2017). Recuperado de http://www.dicc.hegoa.ehu.es/listar/mostrar/52

Diccionario de la Real Academia de la Lengua Española. (2017). Recuperado de http:// dle.rae.es/?id=C1sjxzl

Garay Salamanca, L. J., Barberi Gómez, F., Uprimny Yepes, R., Saffon Sanin, M. P., Prada Pardo, G., Ramírez Gómez, C., \& Forero Álvarez, J. (2009). El reto ante la tragedia humanitaria: reparar de manera integral el despojo de tierras y bienes del desplazamiento forzado. Bogotá, Colombia: Proceso Nacional de Verificación. 
Garay Salamanca, L. J., \& Vargas Valencia, F. (2012). Desafíos de la Restitución de Tierras y Reparación en Colombia. Memoria y Reparación: elementos para una justicia transicional pro víctima. Bogotá, Colombia: Universidad Externado de Colombia.

Gónzalez Morales, F. (2004). El trabajo clínico en materia de Derechos Humanos en América Latina. Bilbao: Universidad de Deusto.

Oficina del Alto Comisionado de las Naciones Unidas para los Derechos Humanos. (2017). Recuperado de http://www.ohchr.org/Documents/Publications/FactSheet29 sp.pdf

Pelletier Quiñones, P. (2012). Estrategias de litigio de interés público en derechos humanos. Revista IIDH. Recuperado de http://historico.juridicas.unam.mx/publica/ librev/rev/iidh/cont/55/pr/pr13.pdf

Unidad de Víctimas. (2017). Unidad de Víctimas. Recuperado de http://rni.unidadvictimas.gov.co/RUV 